\title{
FLOER HOMOLOGY FOR NON-RESONANT MAGNETIC FIELDS ON FLAT TORI
}

\author{
URS FRAUENFELDER, WILL J. MERRY AND GABRIEL P. PATERNAIN
}

\begin{abstract}
In this article we define and compute the Novikov Floer homology associated to a non-resonant magnetic field and a mechanical Hamiltonian on a flat torus $\mathbb{T}^{2 N}$. As a result, we deduce that this Hamiltonian system always has $2 N+1$ contractible solutions, and generically even $2^{2 N}$ contractible solutions. Moreover if there exists a non-degenerate non-contractible solution then there necessarily exists another.
\end{abstract}

\section{INTRODUCTION}

In this paper we study existence and multiplicity results for periodic solutions of fixed period $\tau$ of a system of $N$ interacting particles on the plane subject to a magnetic field. We suppose that the magnetic field as well as the potential are periodic on the plane, so that we can carry out our study on the torus.

Let us first describe our set-up for a single particle, i.e. the case $N=1$. We abbreviate by $\mathbb{T}^{2}=\mathbb{R}^{2} / \mathbb{Z}^{2}$ the two dimensional torus. We model the magnetic field via a 2-form $\sigma \in \Omega^{2}\left(\mathbb{T}^{2}\right)$. If $\pi: T^{*} \mathbb{T}^{2} \rightarrow \mathbb{T}^{2}$ is the footpoint projection, consider the magnetic symplectic form,

$$
\omega_{\sigma}:=d \lambda+\pi^{*} \sigma
$$

where $\lambda=x_{1} d p_{1}+x_{2} d p_{2}$ is the Liouville 1-form on the cotangent bundle of the torus. Abbreviate the circle of length $\tau$ by $\mathbb{S}_{\tau}:=\mathbb{R} / \tau \mathbb{Z}$. For $V \in C^{\infty}\left(\mathbb{S}_{\tau} \times \mathbb{T}^{2}, \mathbb{R}\right)$ a $\tau$-periodic time-dependent potential we define the $\tau$-periodic Hamiltonian $H_{V} \in C^{\infty}\left(\mathbb{S}_{\tau} \times T^{*} \mathbb{T}^{2}, \mathbb{R}\right)$ by

$$
H_{V}(t, x, p)=\frac{1}{2}|p|^{2}+V(t, x)
$$

where the norm of $p$ is taken with respect to the flat metric on the torus. The Hamiltonian vector field $X_{\sigma, V}$ of $H_{V}$ with respect to the magnetic symplectic form is implicitly defined by the equation

$$
\omega_{\sigma}\left(X_{\sigma, V}, \cdot\right)=-d H_{V}
$$

We are interested in $\tau$-periodic solutions of $X_{\sigma, V}$, i.e. solutions $w \in C^{\infty}\left(\mathbb{S}_{\tau}, T^{*} \mathbb{T}^{2}\right)$ of the ODE

$$
\partial_{t} w=X_{\sigma, V}(t, w) \text {. }
$$

We denote by $\mathcal{P}_{\tau}(\sigma, V)$ the moduli space of solutions. Since the fundamental group of the two dimensional torus equals $\pi_{1}\left(\mathbb{T}^{2}\right)=\pi_{1}\left(T^{*} \mathbb{T}^{2}\right)=\mathbb{Z}^{2}$ we get a decomposition of this moduli space as

$$
\mathcal{P}_{\tau}(\sigma, V)=\bigcup_{h \in \mathbb{Z}^{2}} \mathcal{P}_{\tau}^{h}(\sigma, V)
$$

where $\mathcal{P}_{\tau}^{h}(\sigma, V)$ denotes those elements in $\mathcal{P}_{\tau}(\sigma, V)$ which represent $h$ in the fundamental group of $T^{*} \mathbb{T}^{2}$. In particular, $\mathcal{P}_{\tau}^{0}(\sigma, V)$ denotes the contractible solutions.

We do not know if periodic solutions always exist. However, we can prove their existence under the following non-resonance condition we explain next. Denote by $\mu=d x_{1} \wedge d x_{2}$ the 
volume form on the two torus with respect to the flat metric. Then we can write $\sigma=a \mu$ for some function $a \in C^{\infty}\left(\mathbb{T}^{2}, \mathbb{R}\right)$.

Definition 1.1. We say that $\sigma$ is non-resonant in period $\tau$, if there exists $k \in \mathbb{Z}$ such that

$$
\frac{2 \pi k}{\tau}<a(x)<\frac{2 \pi(k+1)}{\tau}, \quad \forall x \in \mathbb{T}^{2} .
$$

Our first main result for a single particle system is the following.

Theorem A. Assume that $\sigma \in \Omega^{2}\left(\mathbb{T}^{2}\right)$ is non-resonant in period $\tau$. Then for every $V \in C^{\infty}\left(\mathbb{S}_{\tau} \times\right.$ $\left.\mathbb{T}^{2}, \mathbb{R}\right)$ we have $\# \mathcal{P}_{\tau}^{0}(\sigma, V) \geq 3$. Moreover, for a generic potential $V$ it holds that $\# \mathcal{P}_{\tau}^{0}(\sigma, V) \geq 4$.

In general noncontractible solutions may not necessarily exist. However, our second main result tells us that if a noncontractible and nondegenerate solution exists a second noncontractible one has to exist as well. For that recall that if $\phi_{\sigma, V}^{t}$ denotes the flow of the Hamiltonian vector field $X_{\sigma, V}$ and $w \in \mathcal{P}_{\tau}(\sigma, V)$, then $w$ is called nondegenerate if

$$
\operatorname{det}\left(D \phi_{\sigma, V}^{\tau}(w(0))-\mathrm{Id}\right) \neq 0 .
$$

Now we are in position to state our next main result for a single particle system.

Theorem B. Assume that $\sigma \in \Omega^{2}\left(\mathbb{T}^{2}\right)$ is a non-resonant magnetic field in period $\tau, V \in C^{\infty}\left(\mathbb{S}_{\tau} \times\right.$ $\left.\mathbb{T}^{2}, \mathbb{R}\right)$, and $w \in \mathcal{P}_{\tau}^{h}(\sigma, V)$ is nondegenerate for some $h \neq 0 \in \pi_{1}\left(\mathbb{T}^{2}\right)$. Then it holds that $\# \mathcal{P}_{\tau}^{h}(\sigma, V) \geq$ 2 .

We next explain how Theorem A and Theorem B generalize to systems of $N$ interacting particles. The configuration space of $N$ particles is given by the $2 N$-dimensional torus $\mathbb{T}^{2 N}$. To keep track of our particles we choose for $1 \leq j \leq N$ a torus $\mathbb{T}_{j}^{2}=\mathbb{T}^{2}$ and think of the $2 N$ dimensional torus as $\mathbb{T}^{2 N}=\prod_{j=1}^{N} \mathbb{T}_{j}^{2}$. We denote by $\mathrm{p}_{j}: \mathbb{T}^{2 N} \rightarrow \mathbb{T}_{j}^{2}$ the canonical projection. We generalize the notion of a non-resonant magnetic field to the many particle case as follows.

Definition 1.2. A two form $\sigma \in \Omega^{2}\left(\mathbb{T}^{2 N}\right)$ is called non-resonant in period $\tau$, if for $1 \leq j \leq N$ there exist non-resonant in period $\tau$ forms $\sigma_{j} \in \Omega^{2}\left(\mathbb{T}_{j}^{2}\right)$ in the sense of Definition 1.1 such that $\sigma=\sum_{j=1}^{N} \mathrm{p}_{j}^{*} \sigma_{j}$.

We are now in position to generalize Theorem A to the case of $N$ particles.

Theorem A+. Assume that $\sigma \in \Omega^{2}\left(\mathbb{T}^{2 N}\right)$ is non-resonant in period $\tau$. Then for every $V \in C^{\infty}\left(\mathbb{S}_{\tau} \times\right.$ $\left.\mathbb{T}^{2 N}, \mathbb{R}\right)$ we have $\# \mathcal{P}_{\tau}^{0}(\sigma, V) \geq 2 N+1$. Moreover, for a generic potential $V$ it holds that $\# \mathcal{P}_{\tau}^{0}(\sigma, V) \geq$ $2^{2 N}$

Of course Theorem A+ immediately implies Theorem A by specializing to the case $N=1$. On the other hand Theorem A+ is not a consequence of Theorem A. Here the key point is that in Theorem A+ we do not need to assume that the potential is of product form, which means that our particles are allowed to interact with each other. In the non interacting case, i.e. the case where the potential $V$ can be written as $V=\sum_{j=1}^{N} \mathrm{p}_{j}^{*} V_{j}$ for potentials $V_{j} \in C^{\infty}\left(\mathbb{S}_{\tau} \times \mathbb{T}_{j}^{2}, \mathbb{R}\right)$ Theorem $\mathrm{A}+$ is an immediate consequence of Theorem A. In fact, in this case we get even the stronger lower bound $3^{N}$ for the number of solutions.

Similarly, Theorem B generalizes to the case of $N$ particles as follows. 
Theorem B+. Assume that $\sigma \in \Omega^{2}\left(\mathbb{T}^{2 N}\right)$ is a non-resonant magnetic field in period $\tau, V \in C^{\infty}\left(\mathbb{S}_{\tau} \times\right.$ $\left.\mathbb{T}^{2 N}, \mathbb{R}\right)$, and $w \in \mathcal{P}_{\tau}^{h}(\sigma, V)$ is nondegenerate for some $h \neq 0 \in \pi_{1}\left(\mathbb{T}^{2 N}\right)$. Then it holds that $\# \mathcal{P}_{\tau}^{h}(\sigma, V) \geq 2$.

We conclude this Introduction by briefly explaining our approach. Unsurprisingly, we use a variant of Floer homology. This semi-infinite dimensional Morse homology associates to this Hamiltonian system a chain complex $C F_{*}^{h}(\sigma, V, \tau)$ which is generated by the elements of $\mathcal{P}_{\tau}^{h}(\sigma, V)$ and defines a boundary operator by counting perturbed holomorphic cylinders which asymptotically converge to the periodic orbits. A priori it is far form obvious that this recipe gives a well defined boundary operator. Indeed, the question as to whether Floer's boundary operator is well-defined or not depends on a difficult compactness result for the perturbed holomorphic curve equation, which cannot be expected to be true in full generality. The main point of the present paper is that under the non-resonant condition from Definition 1.1 we can establish this compactness result. The usefulness of Floer homology stems from its invariance under perturbations. If the magnetic field is a constant multiple $a_{0} \mu$ of the volume form $\mu$ (cf. Definition 1.1) and $\tau>0$ and $k \in\{0,1,2, \ldots$,$\} are such that$

$$
2 \pi k<\tau\left|a_{0}\right|<2 \pi(k+1),
$$

then for the special case $V \equiv 0$ we can directly compute the Floer homology $H F_{*}^{h}\left(a_{0} \mu, 0, \tau\right)$ :

$$
H F_{*}^{h}\left(a_{0} \mu, 0, \tau\right)= \begin{cases}H_{*+2 k}\left(\mathbb{T}^{2} ; \mathbb{Z}\right), & h=0, \\ 0, & h \neq 0 .\end{cases}
$$

Theorems A and B are immediate consequences of (1.2) and the aforementioned invariance properties of $H F_{*}^{h}$. Strictly speaking the case $V \equiv 0$ is never non-degenerate. It is however "weakly non-degenerate" in the sense that the Hamiltonian action functional is Morse-Bott. In this case it is still possible to define the Floer homology; see Section 4 for more information. Theorems $\mathrm{A}+$ and $\mathrm{B}+$ follow via a similar argument.

On a more technical level, there are two additional points of interest in our construction.

Remark 1.3. The symplectic form $\omega_{\sigma}$ is not atoroidal. This means that the Hamiltonian action functional (cf. (3.8)) is not real valued on the loop space of $T^{*} \mathbb{T}^{n}$, and one needs to use a suitable Novikov cover. Thus the Floer homology $H F_{*}^{h}$ that we construct is actually a Novikov Floer homology.

Remark 1.4. As is well known, an alternative (more classical) approach to proving results like Theorems A and B is the Lagrangian formulation where one studies a Lagrangian action functional on the (completed) loop space of $\mathbb{T}^{2}$, and attempts to define a Morse homology for it. When defined, this Morse homology should agree with the Floer homology. For the flavours of Morse/Floer homology we use in this paper this result is due to Abbondandolo and Schwarz [AS06] and is explained in more detail in this setting in [FMP12, Appendix A]. In Section 5 we explain how the non-resonance condition from Definition 1.1 implies that the Lagrangian action functional satisfies the Palais-Smale condition. If $\sigma$ is sufficiently small (more precisely, if one can take $k=0$ in Definition 1.1) then the Lagrangian action functional is also bounded below, and in this case one can recover Theorems A and B using this functional (see e.g. [AM06]). However if $k>0$ in Definition 1.1 then the Lagrangian action functional is no longer bounded below, and hence one cannot define a Morse homology with it. In fact, (1.2) shows that for 
$k>0$, the Floer homology is not the homology of a topological space (it is zero in degree zero) and hence one should not expect to be able to define a Morse homology for the Lagrangian action functional.

The results of this paper were announced in our earlier article [FMP12].

Acknowledgement. We are grateful to Felix Schlenk for his helpful comments and discussions. UF is supported by the Alexander von Humboldt Foundation and by the Basic Research fund 2010-0007669 funded by the Korean government. WM is supported by an ETH Postdoctoral Fellowship.

\section{PRELIMINARIES}

We think of $\mathbb{T}^{2 N}$ as being embedded inside $\mathbb{C}^{2 N}$ as $S^{1} \times \cdots \times S^{1}$. We denote by $\langle\cdot, \cdot\rangle$ the standard Euclidean flat metric on $\mathbb{T}^{2 N}$, which comes from the real part of the Hermitian inner product on $\mathbb{C}^{2 N}$, and we denote by

$$
j=\left(\begin{array}{ll} 
& -\mathrm{Id} \\
\mathrm{Id} &
\end{array}\right)
$$

the canonical almost complex structure on $\mathbb{C}^{2 N}$.

The embedding $\mathbb{T}^{2 N} \subset \mathbb{C}^{2 N}$ induces an embedding of the trivial bundle $T^{*} \mathbb{T}^{2 N}$ inside $\mathbb{C}^{4 N}$. To minimize ambiguity, we write $\langle\langle\cdot, \cdot\rangle\rangle$ for the induced metric on $T^{*} \mathbb{T}^{2 N}$ and $\mathrm{J}$ for the corresponding almost complex structure on $T^{*} \mathbb{T}^{2 N}$ (thus $\langle\langle\cdot, \cdot\rangle\rangle$ and $\mathrm{J}$ are defined in the same way as $\langle\cdot, \cdot\rangle$ and $j$, but on $\mathbb{C}^{4 N}$ instead of $\left.\mathbb{C}^{2 N}\right)$. We will freely use the "musical" isomorphism $v \mapsto\langle v, \cdot\rangle$ to identify $T \mathbb{T}^{2 N}$ with $T^{*} \mathbb{T}^{2 N}$ without further comment. Under this identification $\langle\langle\cdot, \cdot\rangle\rangle$ is the Sasaki metric on $T \mathbb{T}^{2 N}$.

In addition to the isometry $T \mathbb{T}^{2 N} \cong T^{*} \mathbb{T}^{2 N}$, the metric also determines a horizontal-vertical splitting of the tangent bundle $T T^{*} \mathbb{T}^{2 N}$ :

$$
T_{(x, p)} T^{*} \mathbb{T}^{2 N} \cong T_{x} \mathbb{T}^{2 N} \oplus T_{x}^{*} \mathbb{T}^{2 N}
$$

which sends a tangent vector $\xi$ to the pair $\xi \mapsto(D \pi(\xi), K(\xi))$; here $K$ is the connection map associated to the Levi-Civita connection $\nabla$ induced from $\langle\cdot, \cdot\rangle$. In fact, under our embedding this corresponds to the splitting $\mathbb{C}^{4 N} \cong \mathbb{C}^{2 N} \oplus \mathbb{C}^{2 N}$. In particular, if we write $\xi^{h}:=D \pi(\xi)$ and $\xi^{v}:=K(\xi)$ then one has

$$
\langle\langle\xi, \zeta\rangle\rangle=\left\langle\xi^{h}, \zeta^{h}\right\rangle+\left\langle\xi^{v}, \zeta^{v}\right\rangle
$$

Using this notation, the almost complex structure $\mathrm{J}$ sends $\left(\xi^{h}, \xi^{v}\right) \mapsto\left(-\xi^{v}, \xi^{h}\right)$. The almost complex structure $\mathrm{J}$ is compatible with the standard symplectic structure $d \lambda$ in the sense that

$$
d \lambda(\mathrm{J} \cdot, \cdot)=\langle\langle\cdot, \cdot\rangle\rangle .
$$

Now let us introduce a closed 2-form $\sigma$ on $\mathbb{T}^{2 N}$. Whilst all of what follows in this section is valid for any closed 2-form $\sigma$, since in this article we will only work with product 2-forms $\sigma=\sum_{j=1}^{N} \mathbf{p}_{j}^{*} \sigma_{j}$, where each $\sigma_{j} \in \Omega^{2}\left(\mathbb{T}^{2}\right)$, we shall restrict to this case right away. Such a 2-form $\sigma$ is equivalent to an $N$-tuple $\left(a_{1}, \ldots, a_{N}\right)$ of smooth functions $a_{j}: \mathbb{T}^{2} \rightarrow \mathbb{R}$, where $\sigma_{j}=a_{j} \mu$. Here $\mu$ is the volume form on $\mathbb{T}^{2}$ induced by $\langle\cdot, \cdot\rangle$.

The metric $\langle\cdot, \cdot\rangle$ allows us to associate to $\sigma$ an endomorphism $Y_{\sigma}$ of $T \mathbb{T}^{2 N}$, called the Lorentz force, via

$$
\sigma_{x}\left(v, v^{\prime}\right)=\left\langle Y_{\sigma}(x) \cdot v, v^{\prime}\right\rangle
$$


We denote by $\mathcal{J}_{\sigma}$ the open set of almost complex structures $J$ on $T^{*} \mathbb{T}^{2 N}$ that are both compatible with $d \lambda$ and tamed by $\omega_{\sigma}$ - this just means that the bilinear form $d \lambda(J ., \cdot)$ is both positive definite and symmetric, and the bilinear form $\omega_{\sigma}(J \cdot, \cdot)$ is positive definite (but not necessarily symmetric). Unfortunately in general J will not belong to $\mathcal{J}_{\sigma}$. This can be rectified by rescaling. Given $A>0$ we define the rescaled almost complex structure

$$
\mathrm{J}_{A}:=\left(A \cdot \mathrm{Id}{ }^{-\frac{1}{A} \cdot \mathrm{Id}}\right) .
$$

Definition 2.1. Fix $A, \varepsilon>0$. Let $\mathcal{U}(\sigma, A, \varepsilon)$ denote the set of almost complex structures $J \in \mathcal{J}_{\sigma}$ which are uniformly tame in the sense that

$$
\omega_{\sigma}(J \xi, \xi)>\frac{1}{4} d \lambda(J \xi, \xi) \text { for all } \xi \in T T^{*} \mathbb{T}^{2 N},
$$

and which satisfy

$$
\left\|J-\mathrm{J}_{A}\right\|_{\infty}<\varepsilon .
$$

A priori there is no reason why $\mathcal{U}(\sigma, A, \varepsilon)$ should be non-empty. However in [FMP12, Lemma 2], we proved:

Lemma 2.2. If $\sigma=\sum_{j=1}^{N} \mathrm{p}_{j}^{*}\left(a_{j} \mu\right)$ and $A \geq \max _{1 \leq j \leq N}\left\|a_{j}\right\|_{\infty}$ then for any $0<\varepsilon<\frac{1}{7}$ the set $\mathcal{U}(\sigma, A, \varepsilon)$ is open and non-empty. More precisely, one has

$$
B\left(\mathrm{~J}_{A}, \varepsilon\right) \subset \mathcal{U}(\sigma, A, \varepsilon),
$$

where $B\left(\mathrm{~J}_{A}, \varepsilon\right):=\left\{J \in \mathcal{J}_{\sigma} \mid\left\|J-\mathrm{J}_{A}\right\|_{\infty}<\varepsilon\right\}$.

We will also need the following easy result.

Lemma 2.3. Suppose $J$ is an almost complex structure on $T^{*} \mathbb{T}^{2 N}$ which is compatible with $d \lambda$. Let $\kappa_{J}$ denote the minimal positive eigenvalue of the positive symmetric operator $-\mathrm{J} \circ J$. Then for any $v \in T T^{*} \mathbb{T}^{2 N}$ one has

$$
\langle\langle v, v\rangle\rangle \leq \frac{1}{\kappa_{J}} d \lambda(J v, v) .
$$

Proof. A simple computation:

$$
\begin{aligned}
d \lambda(J v, v) & =d \lambda(\mathrm{J} J v, \mathrm{~J} v) \\
& =\langle\langle J v, \mathrm{~J} v\rangle\rangle \\
& =\langle\langle-\mathrm{J} \circ J v, v\rangle\rangle \\
& \geq \kappa_{J}\langle\langle v, v\rangle\rangle .
\end{aligned}
$$

Given $\tau>0$ we write $\mathcal{L}_{\tau} \mathbb{T}^{2 N}=C^{\infty}\left(\mathbb{S}_{\tau}, \mathbb{T}^{2 N}\right)$. For each $h \in \mathbb{Z}^{2 N}=\pi_{1}\left(\mathbb{T}^{2 N}\right)$ we write $\mathcal{L}_{\tau}^{h} \mathbb{T}^{2 N}$ for the component of $\mathcal{L}_{\tau} \mathbb{T}^{2 N}$ of loops belonging to the homotopy class $h$. We write $\mathcal{L}_{\tau} T^{*} \mathbb{T}^{2 N}$ for the free loop space of the cotangent bundle. We define the $L^{2}$-inner products $\langle\cdot, \cdot\rangle_{2}$ on $\mathcal{L}_{\tau} \mathbb{T}^{2 N}$ and $\langle\langle\cdot \cdot \cdot\rangle\rangle_{2}$ on $\mathcal{L}_{\tau} T^{*} \mathbb{T}^{2 N}$ using the metrics $\langle\cdot, \cdot\rangle$ and $\langle\langle\cdot, \cdot\rangle\rangle$ respectively. Thus

$$
\langle\langle\xi, \zeta\rangle\rangle_{2}:=\int_{0}^{\tau}\langle\langle\xi(t), \zeta(t)\rangle\rangle d t .
$$

Let us now rephrase the non-resonance condition in a way that will be more convenient later on. Given $\gamma \in \mathcal{L}_{\tau} \mathbb{T}^{2 N}$, we denote by $F_{\sigma}^{\gamma} \in \Gamma\left(\gamma^{*} \operatorname{End}\left(T \mathbb{T}^{2 N}\right)\right)$ the unique solution to the Cauchy 
problem

$$
\nabla_{t} F_{\sigma}^{\gamma}(t)=F_{\sigma}^{\gamma}(t) \circ Y_{\sigma}(\gamma(t)), \quad F_{\sigma}^{\gamma}(0)=\text { Id } .
$$

Since $Y_{\sigma}$ is antisymmetric, the operator $F_{\sigma}^{\gamma}$ is an orthogonal transformation. If $\sigma=\sum_{j=1}^{N} \mathrm{p}_{j}^{*}\left(a_{j} \mu\right)$ and

$$
b_{j}^{\gamma}(t):=\int_{0}^{t} a_{j}\left(\mathrm{p}_{j}(\gamma(s))\right) d s
$$

then one has for $\left(v_{1}, \ldots, v_{2 N}\right) \in T \mathbb{T}^{2 N}$ that

$$
F_{\sigma}^{\gamma}(t)\left(v_{1}, \ldots, v_{2 N}\right)=\left(\exp \left(b_{1}^{\gamma}(t) \mathrm{j}\right)\left(v_{1}, v_{2}\right), \ldots, \exp \left(b_{N}^{\gamma}(t) \mathrm{j}\right)\left(v_{2 N-1}, v_{2 N}\right)\right)
$$

where here $\mathrm{j}$ is the complex structure on $\mathbb{T}^{2}$ (not $\mathbb{T}^{2 N}$ ), and each pair $\left(v_{2 j-1}, v_{2 j}\right)$ is thought of as belonging to $T \mathbb{T}^{2}$. The following lemma is therefore straightforward.

Lemma 2.4. Suppose $\sigma=\sum_{j=1}^{N} \mathrm{p}_{j}^{*} \sigma_{j}$. Then $\sigma$ is non-resonant in period $\tau$ (in the sense of Definitions 1.1 and 1.2) if and only if there exists $\varepsilon>0$ such that for any curve $\gamma \in \mathcal{L}_{\tau} \mathbb{T}^{2 N}$ and all $v \in T_{\gamma(\tau)} \mathbb{T}^{2 N}$, one has

$$
\left|\left(F_{\sigma}^{\gamma}(\tau)-\mathrm{Id}\right) v\right| \geq \varepsilon|v| .
$$

Remark 2.5. Of course, one can still define $F_{\sigma}^{\gamma}$ even when $\sigma$ is not of the form $\sum_{j=1}^{N} \mathrm{p}_{j}^{*} \sigma_{j}$. The construction in Section 3 would go through without any changes if we took (2.4) as the definition of non-resonance rather than Definitions 1.1 and 1.2, which thus allows us to define the Floer homology groups $H F_{*}^{h}(\sigma, V, \tau)$ for magnetic forms that are not of the form $\sigma=\sum_{j=1}^{N} \mathrm{p}_{j}^{*} \sigma_{j}$. Nevertheless, we do not know how to calculate $H F_{*}^{h}(\sigma, V, \tau)$ unless $\sigma$ is of this form, which is why Theorem A+ and Theorem B+ are stated for product magnetic forms.

\section{Defining the Novikov Floer homology}

3.1. The Novikov framework. Consider the 1 -form $a_{\sigma} \in \Omega^{1}\left(\mathcal{L}_{\tau} \mathbb{T}^{2 N}\right)$ defined by

$$
a_{\sigma}(\gamma)(\xi):=\int_{\mathbb{S}_{\tau}} \sigma\left(\partial_{t} \gamma, \xi\right) d t
$$

Since $\sigma$ is closed, $a_{\sigma}$ is closed, and thus for each $h \in \mathbb{Z}^{2 N} \cong \pi_{1}\left(\mathbb{T}^{2 N}\right)$ we can define a map $\Phi_{\sigma}^{h}: \pi_{1}\left(\mathcal{L}_{\tau}^{h} \mathbb{T}^{2 N}\right) \rightarrow \mathbb{R}$ by

$$
\Phi_{\sigma}^{\alpha}([f]):=\int_{S^{1}} f^{*} a_{\sigma}=\int_{S^{1} \times \mathbb{S}_{\tau}} f^{*} \sigma
$$

where we think of a map $f: S^{1} \rightarrow \mathcal{L}_{\tau}^{h} \mathbb{T}^{2 N}$ representing an element $[f] \in \pi_{1}\left(\mathcal{L}_{\tau}^{h} \mathbb{T}^{2 N}\right)$ also as a $\operatorname{map} f: S^{1} \times \mathbb{S}_{\tau} \rightarrow \mathbb{T}^{2 N}$.

Given $h \in \mathbb{Z}^{2 N}$, thought of as a lattice in $\mathbb{R}^{2 N}$, let $\widetilde{\gamma}_{h}:[0,1] \rightarrow \mathbb{R}^{2 N}$ be defined by

$$
\widetilde{\gamma}_{h}(t):=t h .
$$

Let $\gamma_{h} \in \mathcal{L}_{1}^{h} \mathbb{T}^{2 N}$ denote the projection $\gamma_{h}=q \circ \widetilde{\gamma}_{h}$, where $\mathrm{q}: \mathbb{R}^{2 N} \rightarrow \mathbb{T}^{2 N}$ denotes the universal cover. Denote by

$$
\Gamma_{\sigma}^{h}:=\pi_{1}\left(\mathcal{L}_{\tau}^{h} \mathbb{T}^{2 N}\right) / \operatorname{ker}\left(\Phi_{\sigma}^{h}\right) .
$$

Then $\Gamma_{\sigma}^{h}$ is a finitely generated free abelian group, $\Gamma_{\sigma}^{h} \cong \mathbb{Z}^{m}$ for some $m$. $\Gamma_{\sigma}^{h}$ is cyclic if and only if $\left[a_{\sigma}\right] \in H^{1}\left(\mathcal{L}_{\tau} \mathbb{T}^{2 N} ; \mathbb{Z}\right)$ is an integral class (see [Far04, Lemma 2.1]), that is, if and only if $\sigma \in H^{2}\left(\mathbb{T}^{2 N} ; \mathbb{Z}\right)$ is integral. Let $Q: \mathbb{L}_{\tau}^{h}\left(\mathbb{T}^{2 N}, \sigma\right) \rightarrow \mathcal{L}_{\tau}^{h} \mathbb{T}^{2 N}$ denote the covering space of $\mathcal{L}_{\tau}^{h} \mathbb{T}^{2 N}$ with deck transformation group $\Gamma_{\sigma}^{h}$. We call $Q$ a finite integration cover for $\sigma$ of $\mathcal{L}_{\tau}^{h} \mathbb{T}^{2 N}$. An element of $\mathbb{L}_{\tau}^{h}\left(\mathbb{T}^{2 N}, \sigma\right)$ is an equivalence class $[\gamma, z]$ of a pair $(\gamma, z)$, where $\gamma \in \mathcal{L}_{\tau}^{h} \mathbb{T}^{2 N}$ and 
$z:[0,1] \times \mathbb{S}_{\tau} \rightarrow \mathbb{T}^{2 N}$ satisfies

$$
z(0, t)=\gamma_{h}(t / \tau), \quad z(1, t)=\gamma(t),
$$

and the equivalence relation is given by

$$
\left(\gamma, z_{0}\right) \sim\left(\gamma, z_{1}\right) \Leftrightarrow\left[z_{0} \sharp z_{1}^{-}\right] \in \operatorname{ker}\left(\Phi_{\sigma}^{h}\right),
$$

where $z_{1}^{-}(s, t):=z_{1}(s,-t)$, and $\left[z_{0} \sharp z_{1}^{-}\right]$denotes the element of $\pi_{1}\left(\mathcal{L}_{\tau}^{h} \mathbb{T}^{2 N}\right)$ containing the map $S^{1} \times \mathbb{S}_{\tau} \rightarrow \mathbb{T}^{n}$ obtained by gluing $z_{0}$ and $z_{1}^{-}$along $\gamma_{h}$.

Remark 3.1. Note for $h=0$ one has $\Phi_{\sigma}^{0} \equiv 0$ since the lift of $\sigma$ to the universal cover $\mathbb{R}^{2 N}$ of $\mathbb{T}^{2 N}$ is necessarily exact, and hence $\int_{S^{2}} f^{*} \sigma=0$ for any map $f: S^{2} \rightarrow \mathbb{T}^{2 N}$. Thus for $h=0$ the space $\mathbb{L}_{\tau}^{0}\left(\mathbb{T}^{2 N}, \sigma\right)$ is exactly $\mathcal{L}_{\tau}^{0} \mathbb{T}^{2 N}$.

The action of $\Gamma_{\sigma}^{h}$ on the fibre $Q^{-1}(\gamma)$ is given by $(f,[\gamma, z]) \mapsto[\gamma, z \sharp f]$, where $f: S^{1} \times \mathbb{S}_{\tau} \rightarrow \mathbb{T}^{2 N}$ is a representative of $f \in \Gamma_{\sigma}^{h}$, and $z \sharp f:[0,1] \times \mathbb{S}_{\tau} \rightarrow \mathbb{T}^{2 N}$ is (a smooth reparametrization of) the map

$$
(z \sharp f)(s, t):= \begin{cases}z(2 r, t), & 0 \leq r \leq 1 / 2, \\ f(2 r-1, t), & 1 / 2 \leq r \leq 1 .\end{cases}
$$

It follows directly from the definition that the one-form $Q^{*} a_{\sigma} \in \Omega^{1}\left(\mathbb{L}_{\tau}^{h}\left(\mathbb{T}^{2 N}, \sigma\right)\right)$ is exact. In fact, if $\mathcal{A}_{\sigma}: \mathbb{L}_{\tau}^{h}\left(\mathbb{T}^{2 N}, \sigma\right) \rightarrow \mathbb{R}$ is defined by

$$
\mathcal{A}_{\sigma}([\gamma, z]):=\int_{[0,1] \times \mathbb{S}_{\tau}} z^{*} \sigma
$$

then we have

$$
Q^{*} a_{\sigma}=d \mathcal{A}_{\sigma}
$$

Note that

$$
\mathcal{A}_{\sigma}([\gamma, z \# f])=\mathcal{A}_{\sigma}([\gamma, z])+\Phi_{\sigma}^{h}(f) .
$$

Let $\Lambda_{\sigma}^{h}$ denote the $\mathbb{Z}_{2}$-Novikov ring

$$
\Lambda_{\sigma}^{h}=\left\{\sum_{k=0}^{\infty} c_{k} f_{k} \mid c_{k} \in \mathbb{Z}_{2}, f_{k} \in \Gamma_{\sigma}^{h}, \Phi_{\sigma}^{h}\left(f_{k}\right) \rightarrow-\infty \text { as } k \rightarrow \infty\right\} ;
$$

The fact that $\Gamma_{\sigma}^{h}$ is a finitely generated free abelian group implies that $\Lambda_{\sigma}^{h}$ is always a field ([HS95, Theorem 4.1]).

Remark 3.2. Note that $\mathbb{L}_{\tau}^{h}\left(\mathbb{T}^{2 N}, \sigma\right)$ and $\Lambda_{\sigma}^{h}$ depend only on the projective line in $H^{2}\left(\mathbb{T}^{2 N} ; \mathbb{R}\right)$ determined by $\sigma$, that is, on $\{t[\sigma]: t \in \mathbb{R} \backslash\{0\}\} \subset H^{2}\left(\mathbb{T}^{2 N} ; \mathbb{R}\right)$. Indeed, it suffices to observe that $\operatorname{ker}\left(\Phi_{t \sigma+d \theta}^{h}\right)=\operatorname{ker}\left(\Phi_{\sigma}^{h}\right)$ for each $t \in \mathbb{R} \backslash\{0\}$ and each $\theta \in \Omega^{1}\left(\mathbb{T}^{2 N}\right)$.

It will be convenient to view these covering spaces also as covering spaces of the free loop space $\mathcal{L}_{\tau}^{h} T^{*} \mathbb{T}^{2 N}$ of $T^{*} \mathbb{T}^{2 N}$. To this end, given $h \in \mathbb{Z}^{2 N}$, we denote by $\widehat{Q}: \mathbb{L}_{\tau}^{h}\left(T^{*} \mathbb{T}^{2 N}, \sigma\right) \rightarrow$ $\mathcal{L}_{\tau}^{h} T^{*} \mathbb{T}^{2 N}$ the cover whose fibre $\widehat{Q}^{-1}(w)$ over $w \in \mathcal{L}_{\tau}^{h} T^{*} \mathbb{T}^{2 N}$ is simply the fibre $Q^{-1}(\pi \circ w)$ for the cover $Q: \mathbb{L}_{\tau}^{h}\left(\mathbb{T}^{2 N}, \sigma\right) \rightarrow \mathcal{L}_{\tau}^{h} \mathbb{T}^{2 N}$ defined above. In other words, $\mathbb{L}_{\tau}^{h}\left(T^{*} \mathbb{T}^{2 N}, \sigma\right)$ consists of equivalence classes $[w, z]$ of pairs $(w, z)$, where $w \in \mathcal{L}_{\tau}^{h} T^{*} \mathbb{T}^{n}$ and $z$ satisfies (3.3) (with $\gamma$ replaced by $\pi \circ w$ ), and the equivalence relation is the same as (3.4). Note that the group of deck transformations of $\mathbb{L}_{\tau}^{h}\left(T^{*} \mathbb{T}^{2 N}, \sigma\right)$ is again $\Gamma_{\sigma}^{h}$, and the action $(f,[w, z]) \rightarrow[w, z \sharp f]$ of $\Gamma_{\sigma}^{f}$ on the fibre $\widehat{Q}^{-1}(w)$ is the same as in (3.5). 
Next, given a smooth potential $V \in C^{\infty}\left(\mathbb{S}_{\tau} \times \mathbb{T}^{2 N}, \mathbb{R}\right)$, let $H_{V} \in C^{\infty}\left(\mathbb{S}_{\tau} \times T^{*} \mathbb{T}^{2 N}, \mathbb{R}\right)$ be defined by

$$
H_{V}(t, x, p)=\frac{1}{2}|p|^{2}+V(t, x) .
$$

We define the Hamiltonian action functional $\mathcal{A}_{V}: \mathcal{L}_{\tau}^{h} T^{*} \mathbb{T}^{2 N} \rightarrow \mathbb{R}$ by

$$
\mathcal{A}_{V}(w):=\int_{\mathbb{S}_{\tau}} w^{*} \lambda-\int_{0}^{\tau} H_{V}(t, w) d t
$$

It is well known that the set of critical points of $\mathcal{A}_{V}$ corresponds precisely to the set $\mathcal{P}_{\tau}(\sigma=$ $0, V$ ) of solutions to the ODE (1.1) (with $\sigma=0$ ). Fix $h \in \mathbb{Z}^{2 N}$, and define

$$
\mathcal{A}_{\sigma, V}: \mathbb{L}_{\tau}^{h}\left(T^{*} \mathbb{T}^{2 N}, \sigma\right) \rightarrow \mathbb{R}
$$

by

$$
\begin{aligned}
\mathcal{A}_{\sigma, V}([w, z]) & :=\mathcal{A}_{V}(\widehat{Q}([w, z]))+\mathcal{A}_{\sigma}([\pi \circ w, z]) \\
& =\int_{\mathbb{S}_{\tau}} w^{*} \lambda-\int_{0}^{\tau} H_{V}(t, w) d t+\int_{[0,1] \times \mathbb{S}_{\tau}} z^{*} \sigma .
\end{aligned}
$$

Then

$$
\mathcal{A}_{\sigma, V}([w, z \sharp f])=\mathcal{A}_{\sigma, V}([w, z])+\Phi_{\sigma}^{h}(f), \text { for } f \in \Gamma_{\sigma}^{h},
$$

and it is not hard to check that the set $\operatorname{Crit}_{\tau}^{h}\left(\mathcal{A}_{\sigma, V}\right)$ of critical points of $\mathcal{A}_{\sigma, V}$ on $\mathbb{L}_{\tau}^{h}\left(T^{*} \mathbb{T}^{2 N}, \sigma\right)$ is simply the preimage $\widehat{Q}^{-1}\left(\mathcal{P}_{\tau}^{h}(\sigma, V)\right)$ :

$$
\operatorname{Crit}_{\tau}^{h}\left(\mathcal{A}_{\sigma, V}\right)=\left\{[w, z] \in \mathbb{L}_{\tau}^{h}\left(\mathbb{T}^{2 N}, \sigma\right) \mid w \in \mathcal{P}_{\tau}^{h}(\sigma, V)\right\} .
$$

Recall that a critical point $[w, z]$ of $\mathcal{A}_{\sigma, V}$ is said to be non-degenerate if

$$
\operatorname{det}\left(D \phi_{\sigma, V}^{\tau}(w(0))-\mathrm{Id}\right) \neq 0,
$$

where $\phi_{\sigma, V}^{t}$ denotes the flow of $X_{\sigma, V}$.

Definition 3.3. We say that the triple $(\sigma, V, \tau)$ is non-degenerate if every element of $\operatorname{Crit}_{\tau}^{h}\left(\mathcal{A}_{\sigma, V}\right)$ is non-degenerate (for all $h \in \mathbb{Z}^{2 N}$ ). Equivalently, $(\sigma, V, \tau)$ is non-degenerate if and only if $\mathcal{A}_{\sigma, V}: \mathbb{L}_{\tau}^{h}\left(T^{*} \mathbb{T}^{2 N}, \sigma\right) \rightarrow \mathbb{R}$ is a Morse function for all $h \in \mathbb{Z}^{2 N}$.

The next result is very standard; a proof for $\sigma=0$ can be found in [Web02, Theorem 1.1], and the same argument goes through with only minor changes in the general case.

Theorem 3.4. For a given closed 2-form $\sigma \in \Omega^{2}\left(\mathbb{T}^{2 N}\right)$ and a given $\tau>0$, the set of potentials $V \in C^{\infty}\left(\mathbb{S}_{\tau} \times \mathbb{T}^{2 N}, \mathbb{R}\right)$ for which $(\sigma, V, \tau)$ is non-degenerate, is of second category in $C^{\infty}\left(\mathbb{S}_{\tau} \times \mathbb{T}^{2 N}, \mathbb{R}\right)$.

Set

$$
\mathcal{F}_{\tau}^{h}(\sigma, V ; \delta):=\left\{w \in \mathcal{L}_{\tau}^{h} T^{*} \mathbb{T}^{2 N} \mid\left\|\partial_{t} w-X_{\sigma, V}(t, w)\right\|_{2} \leq \delta\right\} .
$$

The following lemma explains why the non-resonance condition from Definition 1.1 is important.

Lemma 3.5. Assume $\sigma$ is non-resonant in period $\tau$, and let $\varepsilon>0$ be such that (2.4) holds. Then for all $w=(\gamma, p) \in \mathcal{F}_{\tau}^{h}(\sigma, V ; \delta)$ one has

$$
\|p\|_{\infty} \leq\left(\sqrt{\tau}+\frac{\sqrt{2 \tau}}{\varepsilon}\right)\left(\delta+\sqrt{\tau}\|\nabla V\|_{\infty}\right) .
$$


Proof. In terms of the horizontal vertical splitting (2.1) one has

$$
\partial_{t} w-X_{\sigma, V}(t, w)=\left(\begin{array}{c}
\partial_{t} \gamma-p \\
\nabla_{t} p+Y_{\sigma}(\gamma) p+\nabla V_{t}(\gamma)
\end{array}\right)
$$

and hence

$$
\left\|\partial_{t} w-X_{\sigma, V}(t, w)\right\|_{2}^{2}=\int_{0}^{\tau}\left|\nabla_{t} p+Y_{\sigma}(\gamma) p+\nabla V_{t}(\gamma)\right|^{2} d t+\int_{0}^{\tau}\left|\partial_{t} \gamma-p\right|^{2} d t .
$$

Fix $w \in \mathcal{F}_{\tau}^{h}(\sigma, V ; \delta)$. Since $T^{*} \mathbb{T}^{2 N} \cong \mathbb{T}^{2 N} \times \mathbb{R}^{2 N}$ is a trivial vector bundle, it makes sense to consider the path $y=(\gamma(0), \eta):[0, \tau] \rightarrow T^{*} \mathbb{T}^{2 N}$ by

$$
\eta(t):=F_{\sigma}^{\gamma}(t) \cdot p(t),
$$

where $F_{\sigma}^{\gamma}(t)$ was defined in (2.3). Observe that

$$
\begin{aligned}
\nabla_{t} \eta & =\left(\nabla_{t} F_{\sigma}^{\gamma}\right) \cdot p+F_{\sigma}^{\gamma} \cdot \nabla_{t} p \\
& =F_{\sigma}^{\gamma}\left(\nabla_{t} p+Y_{\sigma}(\gamma) p\right),
\end{aligned}
$$

Since $F_{\sigma}^{\gamma}(t)$ is an orthogonal transformation of $T_{\gamma(t)} \mathbb{T}^{2 N}$, we have

$$
\begin{aligned}
\int_{0}^{\tau}\left|\nabla_{t} \eta(t)\right|^{2} d t & =\int_{0}^{\tau}\left|\nabla_{t} p+Y_{\sigma}(\gamma) p\right|^{2} d t \\
& \leq 2\left(\int_{0}^{\tau}\left|\nabla_{t} p+Y_{\sigma}(\gamma) p+\nabla V_{t}(\gamma)\right|^{2} d t+\int_{0}^{\tau}\left|\nabla V_{t}(\gamma)\right|^{2} d t\right) \\
& \leq 2 \delta^{2}+2 \tau\|\nabla V\|_{\infty}^{2},
\end{aligned}
$$

where we used $a^{2} \leq 2(a+b)^{2}+2 b^{2}$. Thus in particular,

$$
\operatorname{dist}(y(0), y(\tau)) \leq \int_{0}^{\tau}\left|\nabla_{t} \eta(t)\right| d t \leq \sqrt{\tau} \cdot \sqrt{2 \delta^{2}+2 \tau\|\nabla V\|_{\infty}^{2}},
$$

where dist is the distance measured with with respect to the metric $\langle\langle\cdot, \cdot\rangle\rangle$ on $T \mathbb{T}^{2 N}$. However since

$$
\eta(0)=p(0), \quad \eta(\tau)=F_{\sigma}^{\gamma}(\tau) \cdot p(\tau)
$$

one has

$$
\operatorname{dist}(y(0), y(\tau)) \geq\left|\left(F_{\sigma}^{\gamma}(\tau)-\mathrm{Id}\right) p(0)\right| \geq \varepsilon|p(0)|,
$$

where we are using the fact that $\langle\cdot, \cdot\rangle$ is flat to conclude that the $\langle\langle\cdot, \cdot\rangle\rangle$-geodesic running from $y(0)=(\gamma(0), \eta(0))$ to $y(\tau)=(\gamma(0), \eta(\tau))$ is the straight line in $T_{\gamma(0)} \mathbb{T}^{n}$ from $\eta(0)$ to $\eta(\tau)$. Combining (3.12) and (3.13) we conclude that

$$
|p(0)| \leq \frac{\sqrt{\tau}}{\varepsilon} \sqrt{2 \delta^{2}+2 \tau\|\nabla V\|_{\infty}^{2}} \leq \frac{\sqrt{2 \tau}}{\varepsilon}\left(\delta+\sqrt{\tau}\|\nabla V\|_{\infty}\right) .
$$


Next, given $s \in \mathbb{S}_{\tau}$ one has

$$
\begin{aligned}
|| p(s)|-| p(0) \| & \leq \int_{0}^{s}\left|\nabla_{t}(|p(t)|)\right| d t \\
& \leq \int_{0}^{\tau} \frac{\left|\left\langle\nabla_{t} p, p\right\rangle\right|}{|p|} d t \\
& \stackrel{(*)}{=} \int_{0}^{\tau} \frac{\left|\left\langle\nabla_{t} p+Y_{\sigma}(\gamma) p, p\right\rangle\right|}{|p|} d t \\
& \leq \int_{0}^{\tau}\left|\nabla_{t} p+Y_{\sigma}(\gamma) p\right| d t \\
& \leq \int_{0}^{\tau}\left|\nabla_{t} p+Y_{\sigma}(\gamma) p+\nabla V_{t}(\gamma)\right| d t+\int_{0}^{\tau}\left|\nabla V_{t}(\gamma)\right| d t \\
& \leq \sqrt{\tau}\left(\int_{0}^{\tau}\left|\nabla_{t} p+Y_{\sigma}(\gamma) p+\nabla V_{t}(\gamma)\right|^{2} d t\right)^{1 / 2}+\tau\|\nabla V\|_{\infty} \\
& \leq \sqrt{\tau}\left(\delta+\sqrt{\tau}\|\nabla V\|_{\infty}\right),
\end{aligned}
$$

where $(*)$ used the fact that $\left\langle Y_{\sigma}(\gamma) p, p\right\rangle=\sigma_{\gamma}(p, p)=0$. Thus

$$
\max _{t \in \mathbb{S}_{\tau}}|p(t)| \leq\left(\sqrt{\tau}+\frac{\sqrt{2 \tau}}{\varepsilon}\right)\left(\delta+\sqrt{\tau}\|\nabla V\|_{\infty}\right) .
$$

Remark 3.6. The only place in the paper where it is crucial we are working on a torus $\mathbb{T}^{n}$ is in the preceding lemma. More precisely, (3.12) and (3.13) use the fact that $\mathbb{T}^{n}$ is parallelizable and has a flat metric $\langle\cdot, \cdot\rangle$.

Corollary 3.7. Suppose $\sigma$ is non-resonant in period $\tau$. Then for any potential $V \in C^{\infty}\left(\mathbb{S}_{\tau} \times \mathbb{T}^{2 N}, \mathbb{R}\right)$, the set $\mathcal{P}_{\tau}(\sigma, V)$ is compact. Thus if $(\sigma, V, \tau)$ is non-degenerate then $\mathcal{P}_{\tau}(\sigma, V)$ is a finite set.

Remark 3.8. In fact, a similar argument shows that if $\sigma$ is non-resonant in period $\tau$ then $\mathcal{P}_{\tau}(\sigma, 0)$ has no non-constant solutions. Indeed, if $w=(\gamma, p)$ belongs to $\mathcal{P}_{\tau}(\sigma, 0)$ then it follows from (3.10) that $p=0$ and that $\gamma$ is constant (cf. (3.11)). In particular, this shows that $\mathcal{P}_{\tau}^{h}(\sigma, 0)=\emptyset$ for all $h \neq 0$.

As mentioned in Remark 2.5, Lemma 3.5, Corollary 3.7 and Remark 3.8 all go through if we take the validity of 2.4 to be the definition of non-resonance.

3.2. Moduli spaces. From now on we assume that $\sigma$ is non-resonant in period $\tau$, and $(\sigma, V, \tau)$ is non-degenerate. Fix two critical points $\left[w^{-}, z^{-}\right],\left[w^{+}, z^{+}\right] \in \operatorname{Crit}_{\tau}^{h}\left(\mathcal{A}_{\sigma, V}\right)$. Write $\sigma=\sum_{j=1}^{N} \mathbf{p}_{j}^{*}\left(a_{j} \mu\right)$, and choose $A \geq \max _{1 \leq j \leq N}\left\|a_{j}\right\|_{\infty}$. We will work with paths $\left\{J_{t}\right\}_{t \in \mathbb{S}_{\tau}} \subset \mathcal{U}\left(\sigma, A, \varepsilon_{0}\right)$, where the set $\mathcal{U}\left(\sigma, A, \varepsilon_{0}\right)$ was defined in Definition 2.1, and $\varepsilon_{0}>0$ is a small constant to be specified later. Lemma 2.2 shows that such paths $\left\{J_{t}\right\}$ exist, and moreover that a sufficiently small perturbation $\left\{J_{t}^{\prime}\right\}$ of $\left\{J_{t}\right\}$ still belongs to $\mathcal{U}\left(\sigma, A, \varepsilon_{0}\right)$. This means that it makes sense to talk about a "generic" family $\left\{J_{t}\right\} \subset \mathcal{U}(\sigma, A, \varepsilon)$.

Denote by

$$
\mathcal{M}_{\tau}^{h}\left(\left[w^{-}, z^{-}\right],\left[w^{+}, z^{+}\right], \sigma, V,\left\{J_{t}\right\}\right)
$$

the set of smooth maps $u: \mathbb{R} \times \mathbb{S}_{\tau} \rightarrow T^{*} \mathbb{T}^{2 N}$ which satisfy the Floer equation:

$$
\partial_{s} u+J_{t}(u)\left(\partial_{t} u-X_{\sigma, V}(t, u)\right)=0,
$$


and which submit to the asymptotic conditions

$$
\lim _{s \rightarrow \pm \infty} u(s, t)=w^{ \pm}(t), \quad \lim _{s \rightarrow \pm \infty} \partial_{s} u(s, t)=0, \quad \text { uniformly in } t,
$$

and which in addition satisfy

$$
\left[w^{+}, z^{-} \sharp(\pi \circ \bar{u})\right]=\left[w^{+}, z^{+}\right] .
$$

Here $\bar{u}:[0,1] \times \mathbb{S}_{\tau} \rightarrow T^{*} \mathbb{T}^{2 N}$ is the compactification of $u$ (such a map $\bar{u}$ exists as the convergence of $u(s, t)$ to $w^{ \pm}(t)$ as $s \rightarrow \pm \infty$ is exponentially fast due to the assumption that $\left[w^{ \pm}, z^{ \pm}\right]$are nondegenerate), and $z^{-} \sharp(\pi \circ \bar{u})$ denotes (a smooth reparametrization) of the map

$$
\left(z^{-} \sharp(\pi \circ \bar{u})\right)(r, t)= \begin{cases}z^{-}(2 r, t), & 0 \leq r \leq 1 / 2, \\ (\pi \circ \bar{u})(2 r-1, t), & 1 / 2 \leq r \leq 1 .\end{cases}
$$

We can consider $u \in \mathcal{M}_{\tau}^{h}\left(\left[w^{-}, z^{-}\right],\left[w^{+}, z^{+}\right], \sigma, V,\left\{J_{t}\right\}\right)$ as a map $\widehat{u}: \mathbb{R} \rightarrow \mathbb{L}_{\tau}^{h}\left(T^{*} \mathbb{T}^{2 N}, \sigma\right)$ as follows. Write $u(s, t)=(x(s, t), p(s, t))$, and set

$$
\widehat{u}(s):=[u(s, \cdot), z(s)],
$$

where $z(s)$ is (a smooth reparametrization) of the cylinder obtained by gluing $z^{-}$onto the cylinder $\{x(r, t)\}_{(r, t) \in(-\infty, s] \times \mathbb{S}_{\tau}}$. Thus $\widehat{u}$ is a flow line of $\mathcal{A}_{\sigma, V}$ :

$$
\partial_{s} \widehat{u}+\nabla_{J_{t}} \mathcal{A}_{\sigma, V}(\widehat{u}(s))=0,
$$

where $\nabla_{J_{t}} \mathcal{A}_{\sigma, V}$ denotes the vector field on $\mathbb{L}_{\tau}^{h}\left(T^{*} \mathbb{T}^{2 N}, \sigma\right)$ defined by

$$
\nabla_{J_{t}} \mathcal{A}_{\sigma, V}([w, z]):=J_{t}(w)\left(\partial_{t} w-X_{\sigma, V}(t, w)\right) .
$$

Lemma 3.9. If $u \in \mathcal{M}_{\tau}^{h}\left(\left[w^{-}, z^{-}\right],\left[w^{+}, z^{+}\right], \sigma, V,\left\{J_{t}\right\}\right)$ then

$$
\left\|\partial_{s} u\right\|_{2}^{2} \leq 4 \sup _{t \in \mathbb{S}_{\tau}} \frac{1}{\kappa_{J_{t}}}\left(\mathcal{A}_{\sigma, V}\left(\left[w^{-}, z^{-}\right]\right)-\mathcal{A}_{\sigma, V}\left(\left[w^{+}, z^{+}\right]\right)\right.
$$

where $\kappa_{J_{t}}>0$ denote the minimal positive eigenvalue of $-\mathrm{J} \circ J_{t}$, as in Lemma 2.3.

Proof. We compute:

$$
\begin{aligned}
\left\|\partial_{s} u\right\|_{2}^{2} & \stackrel{\text { def }}{=} \int_{-\infty}^{\infty} \int_{0}^{\tau}\left\langle\left\langle\partial_{s} u, \partial_{s} u\right\rangle\right\rangle d t d s \\
& \leq \sup _{t \in \mathbb{S}_{\tau}} \frac{1}{\kappa_{J_{t}}} \int_{-\infty}^{\infty} \int_{0}^{\tau} d \lambda\left(J_{t} \partial_{s} u, \partial_{s} u\right) u d t d s \\
& \leq 4 \sup _{t \in \mathbb{S}_{\tau}} \frac{1}{\kappa_{J_{t}}} \int_{-\infty}^{\infty} \int_{0}^{\tau} \omega_{\sigma}\left(J_{t} \partial_{s} u, \partial_{s} u\right) d t d s \\
& =4 \sup _{t \in \mathbb{S}_{\tau}} \frac{1}{\kappa_{J_{t}}}\left(\lim _{s \rightarrow-\infty} \mathcal{A}_{\sigma, V}(\widehat{u}(s))-\lim _{s \rightarrow \infty} \mathcal{A}_{\sigma, V}(\widehat{u}(s))\right) \\
& =4 \sup _{t \in \mathbb{S}_{\tau}} \frac{1}{\kappa_{J_{t}}}\left(\mathcal{A}_{\sigma, V}\left(\left[w^{-}, z^{-}\right]\right)-\mathcal{A}_{\sigma, V}\left(\left[w^{+}, z^{+}\right]\right)\right) .
\end{aligned}
$$

We now wish to prove that the moduli spaces $\mathcal{M}_{\tau}^{h}\left(\left[w^{-}, z^{-}\right],\left[w^{+}, z^{+}\right], \sigma, V,\left\{J_{t}\right\}\right)$ have good compactness properties. The next result is an easy consequence of Lemma 3.5.

Lemma 3.10. There exists a constant $T>0$ such that if

$$
u=(x, p) \in \mathcal{M}_{\tau}^{h}\left(\left[w^{-}, z^{-}\right],\left[w^{+}, z^{+}\right], \sigma, V,\left\{J_{t}\right\}\right)
$$


then

$$
\|p(s, \cdot)\|_{2} \leq T\left(1+\left\|\partial_{s} u(s, \cdot)\right\|_{2}\right) .
$$

The following theorem is the central result of this section.

Theorem 3.11. There exists $\varepsilon_{0}>0$ such that if $\left\{J_{t}\right\}_{t \in \mathbb{S}_{\tau}} \subset \mathcal{U}\left(\sigma, A, \varepsilon_{0}\right)$ then for any pair $(C, E) \subseteq$ $\mathbb{R} \times[0, \infty)$, there exists a compact set $K(A, C, E) \subseteq T^{*} \mathbb{T}^{2 N}$ with the following property: Suppose $\left[w^{ \pm}, z^{ \pm}\right] \in \operatorname{Crit}_{\tau}^{h}\left(\mathcal{A}_{\sigma, V}\right)$ satisfy

$$
\begin{gathered}
\mathcal{A}_{\sigma, V}\left(\left[w^{-}, z^{-}\right]\right) \leq C, \\
\mathcal{A}_{\sigma, V}\left(\left[w^{-}, z^{-}\right]\right)-\mathcal{A}_{\sigma, V}\left(\left[w^{+}, z^{+}\right]\right) \leq E .
\end{gathered}
$$

Then if $u \in \mathcal{M}_{\tau}^{h}\left(\left[w^{-}, z^{-}\right],\left[w^{+}, z^{+}\right], \sigma, V,\left\{J_{t}\right\}\right)$ one has

$$
u\left(\mathbb{R} \times \mathbb{S}_{\tau}\right) \subseteq K(A, C, E) .
$$

Theorem 3.11 can be proved following Abbondandolo and Schwarz' method in [AS06], as we now explain. The method has two distinct stages. The first stage appears as Lemma 1.12 in [AS06], and asserts that under the hypotheses of the theorem, there exists a constant $S(A, C, E)>0$ such that for any $u=(x, p)$ belonging to $\mathcal{M}_{\tau}^{h}\left(\left[w^{-}, z^{-}\right],\left[w^{+}, z^{+}\right], \sigma, V,\left\{J_{t}\right\}\right)$, and any interval $\mathbb{I} \subseteq \mathbb{R}$ it holds that

$$
\left\|\left.p\right|_{\mathbb{I} \times \mathbb{S}_{\tau}}\right\|_{2} \leq S(C, E)|\mathbb{I}|^{1 / 2}, \quad\left\|\left.\nabla p\right|_{\mathbb{I} \times \mathbb{S}_{\tau}}\right\|_{2} \leq S(C, E)\left(|\mathbb{I}|^{1 / 2}+1\right) .
$$

A careful inspection of their proof shows that everything apart from Claim 1 and Claim 2 goes through verbatim in our case. Claim 1 however is precisely the statement of Lemma 3.9, and Claim 2 is precisely the statement of Lemma 3.10. The second stage appears as Theorem 1.14 in [AS06]. The proof then uses Calderon-Zygmund estimates for the Cauchy-Riemann operator, together with certain interpolation inequalities, to upgrade equation (3.16) to the full statement of Theorem 3.11. It is this stage that requires $\sup _{t \in \mathbb{S}_{\tau}}\left\|J_{t}-\mathrm{J}_{A}\right\|$ to be sufficiently small (for some $A>0$ ), and thus which determines the constant $\varepsilon_{0}>0$ referred to at the start of this section. Anyway, provided that this is satisfied, the proof of this stage goes through word for word in our situation.

3.3. The Novikov chain complex. We continue to assume that $\sigma$ is non-resonant in period $\tau$, and $(\sigma, V, \tau)$ is non-degenerate. For each $w \in \mathcal{P}_{\tau}^{h}(\sigma, V)$, let $\mu_{\mathrm{CZ}}(w)$ denote the Conley-Zehnder index of $w$. In order to define the Conley-Zehnder index we choose a vertical preserving symplectic trivialization (see [AS06]); the fact that $c_{1}\left(T^{*} M, \omega_{\sigma}\right)=0$ means that that the value of $\mu_{\mathrm{CZ}}(w)$ is independent of this choice of trivialization. Note however that our sign conventions match those of [AS10] not [AS06]. The non-degeneracy condition (3.9) implies that $\mu_{\mathrm{CZ}}(w)$ is always an integer.

Given $j \in \mathbb{Z}$ let

$$
\begin{gathered}
\mathcal{P}_{\tau}^{h}(\sigma, V)_{j}:=\left\{w \in \mathcal{P}_{\tau}^{h}(\sigma, V) \mid \mu_{\mathrm{CZ}}(w)=j\right\}, \\
\operatorname{Crit}_{\tau}^{h}\left(\mathcal{A}_{\sigma, V}\right)_{j}:=\left\{[w, z] \in \operatorname{Crit}_{\tau}^{h}\left(\mathcal{A}_{\sigma, V}\right) \mid \mu_{\mathrm{CZ}}(w)=j\right\} .
\end{gathered}
$$

It follows from Theorem 3.11 that for a generic family $\left\{J_{t}\right\}_{t \in \mathbb{S}_{\tau}} \subset \mathcal{U}\left(\sigma, A, \varepsilon_{0}\right)$ the moduli spaces $\mathcal{M}_{\tau}^{h}\left(\left[w^{-}, z^{-}\right],\left[w^{+}, z^{+}\right], \sigma, V,\left\{J_{t}\right\}\right)$ all carry the structure of a $\left(\mu_{\mathrm{CZ}}\left(w^{-}\right)-\mu_{\mathrm{CZ}}\left(w^{+}\right)\right)$-dimensional manifold. Moreover if $\mu_{\mathrm{CZ}}\left(w^{-}\right)=\mu_{\mathrm{CZ}}\left(w^{+}\right)+1$ then the quotient space

$$
\mathcal{M}_{\tau}^{h}\left(\left[w^{-}, z^{-}\right],\left[w^{+}, z^{+}\right], \sigma, V,\left\{J_{t}\right\}\right) / \mathbb{R}
$$


is a finite set. We define the Novikov Floer chain group as

$$
\begin{aligned}
C F_{j}^{h}(\sigma, V, \tau): & =\left\{\sum_{k=0}^{\infty} c_{k}\left[w_{k}, z_{k}\right] \mid \mu_{\mathrm{CZ}}\left(w_{k}\right)=j, \mathcal{A}_{\sigma, V}\left(\left[w_{k}, z_{k}\right]\right) \rightarrow \infty \text { as } k \rightarrow \infty\right\} . \\
& \cong \mathcal{P}_{\tau}^{h}(\sigma, V)_{j} \otimes \Lambda_{\sigma}^{h} .
\end{aligned}
$$

Thus $C F_{j}^{h}(\sigma, V, \tau)$ is a $\# \mathcal{P}_{\tau}^{h}(\sigma, V)_{j}$-dimensional vector space over $\Lambda_{\sigma}^{h}$ (note our assumptions imply $\mathcal{P}_{\tau}^{h}(\sigma, V)$ is a finite set, cf. Corollary 3.7). The boundary operator $\partial_{J_{t}}: C F_{j} \rightarrow C F_{j-1}$ is defined by

$$
\partial_{J_{t}}([w, z]):=\sum_{\left[w^{\prime}, z^{\prime}\right] \in \operatorname{Crit}_{\tau}^{h}\left(\mathcal{A}_{\sigma, V}\right)_{j-1}} n\left([w, z],\left[w^{\prime}, z^{\prime}\right]\right)\left[w^{\prime}, z^{\prime}\right], \quad[w, z] \in \operatorname{Crit}_{\tau}^{h}\left(\mathcal{A}_{\sigma, V}\right)_{j},
$$

where

$$
n\left([w, z],\left[w^{\prime}, z^{\prime}\right]\right):=\#_{2} \mathcal{M}_{\tau}^{h}\left([w, z],\left[w^{\prime}, z^{\prime}\right], \sigma, V,\left\{J_{t}\right\}\right) / \mathbb{R} .
$$

The fact that the boundary operator is well defined (i.e. $\partial_{J_{t}}([w, z])$ is a well defined element of $\left.C F_{j-1}\right)$ is an immediate consequence of Theorem 3.11.

A standard Floer-theoretic argument, as explained in [HS95, Section 5], tells us that $\partial_{J_{t}} \circ$ $\partial_{J_{t}}=0$, and hence we may define the Novikov Floer homology $H F_{*}^{h}(\sigma, V, \tau)$ to be the homology of the chain complex $\left\{C F_{*}^{h}(\sigma, V, \tau), \partial_{J_{t}}\right\}$. Moreover $H F_{*}^{h}(\sigma, V, \tau)$ is independent (up to canonical isomorphism) of the choice family of almost complex structures $\left\{J_{t}\right\}_{t \in \mathbb{S}_{\tau}} \subset \mathcal{U}\left(\sigma, A, \varepsilon_{0}\right)$ (see for instance [AS06, Theorem 1.19]), which explains why we may safely omit it from our notation.

Remark 3.12. Suppose that $\sigma$ is non-resonant in period $\tau$ but that $(\sigma, V, \tau)$ is degenerate. By Theorem 3.4 we can make an arbitrarily small perturbation of the potential $V$ to a new one $V^{\prime}$ such that $\left(\sigma, V^{\prime}, \tau\right)$ is non-degenerate. Moreover if $V^{\prime \prime}$ is another such perturbation then by Theorem 3.13 below we have $H F_{*}^{h}\left(\sigma, V^{\prime}, \tau\right) \cong H F_{*}^{h}\left(\sigma, V^{\prime \prime}, \tau\right)$. In other words, we can still define $H F_{*}^{h}(\sigma, V, \tau)$ even when $(\sigma, V, \tau)$ is degenerate, by simply setting

$$
H F_{*}^{h}(\sigma, V, \tau) \stackrel{\text { def }}{=} H F_{*}^{h}\left(\sigma, V^{\prime}, \tau\right)
$$

for any potential $V^{\prime}$ such that $\left\|V-V^{\prime}\right\|_{\infty}$ is sufficiently small and such that $\left(\sigma, V^{\prime}, \tau\right)$ is nondegenerate.

3.4. Invariance. In this section we prove invariance through exact deformations of the magnetic form $\sigma$, and deformations of the potential $V$. Fix a 1-form $\theta \in \Omega^{1}\left(\mathbb{T}^{2 N}\right)$, and set

$$
\sigma_{s}:=\sigma+s d \theta
$$

Fix $\tau>0$ and assume that:

- $\sigma_{s}$ is non-resonant in period $\tau$ for all $s \in[0,1]$.

Note that $\Lambda_{\sigma_{s}}^{h} \equiv \Lambda_{\sigma}^{h}$ for all $s \in[0,1]$ (cf. Remark 3.2). Suppose $V_{0}, V_{1} \in C^{\infty}\left(\mathbb{S}_{\tau} \times \mathbb{T}^{2 N}, \mathbb{R}\right)$. Set

$$
V_{s}:=(1-s) V_{0}+s V_{1} \text {. }
$$

Assume that:

- For generic $s \in[0,1]$, and in particular for $s=0,1$, all the triples $\left(\sigma_{s}, V_{s}, \tau\right)$ are nondegenerate.

Under these conditions we have proved that the Floer homologies $H F_{*}^{h}\left(\sigma_{0}, V_{0}, \tau\right)$ and $H F_{*}^{h}\left(\sigma_{1}, V_{1}, \tau\right)$ are both well defined. We now wish to prove they are isomorphic. 
Theorem 3.13. Under the above assumptions there exists a continuation map

$$
\Psi: C F_{*}^{h}\left(\sigma_{0}, V_{0}, \tau\right) \rightarrow C F_{*}^{h}\left(\sigma_{1}, V_{1}, \tau\right)
$$

inducing an isomorphism

$$
\psi: H F_{*}^{h}\left(\sigma_{0}, V_{0}, \tau\right) \rightarrow H F_{*}^{h}\left(\sigma_{1}, V_{1}, \tau\right) .
$$

The key ingredient needed to prove Theorem 3.13 is the following proposition, which proves energy estimates for certain $s$-dependent trajectories. Fix a smooth cutoff function $\beta: \mathbb{R} \rightarrow[0,1]$ satisfying $\beta(s) \equiv 0$ for $s \leq 0$ and $\beta(s) \equiv 1$ for $s \geq 1$, with $0 \leq \beta^{\prime}(s) \leq 2$ for all $s \in \mathbb{R}$.

Proposition 3.14. There exists $\delta>0$ with the following property. Suppose $0=s_{0}<s_{1}<\cdots<$ $s_{N}=1$ satisfies

$$
\max _{j}\left(s_{j+1}-s_{j}\right)<\delta
$$

and set

$$
\begin{gathered}
\beta_{j}(s):=s_{j}+\beta(s)\left(s_{j+1}-s_{j}\right), \\
\sigma_{s}^{j}:=\sigma+\beta_{j}(s) d \theta, \quad \sigma^{j}:=\sigma_{s_{j}}, \\
V_{s}^{j}(t, x):=V_{\beta_{j}(s)}(t, x), V^{j}:=V_{s_{j}} .
\end{gathered}
$$

Then given any $E \in \mathbb{R}$ there exists $R(E)>0$ with the following property: for any $j=0,1, \ldots, N-1$, if $\left[w^{-}, z^{-}\right] \in \operatorname{Crit}_{\tau}^{h}\left(\mathcal{A}_{\sigma^{j}, V^{j}}\right)$ and $\left[w^{+}, z^{+}\right] \in \operatorname{Crit}_{\tau}^{h}\left(\mathcal{A}_{\sigma^{j+1}, V^{j+1}}\right)$ satisfy

$$
\mathcal{A}_{\sigma^{j}, V^{j}}\left(\left[w^{-}, z^{+}\right]\right)-\mathcal{A}_{\sigma^{j+1}, V^{j+1}}\left(\left[w^{+}, z^{+}\right]\right) \leq E,
$$

then given any solution

$$
u \in \mathcal{M}_{\tau}^{h}\left(\left[w^{-}, z^{-}\right],\left[w^{+}, z^{+}\right], \sigma_{s}^{j}, V_{s}^{j},\left\{J_{t}\right\}\right)
$$

(where the moduli space of s-dependent solutions is defined analogously to before) it holds that

$$
\left\|\partial_{s} u\right\|_{2}^{2} \leq R(E) .
$$

Proof. Fix $j \in\{0,1, \ldots, N-1\}$. As in Section 3.2, it is convenient to interpret a map $u(s, t)=$ $(x(s, t), p(s, t))$ as in the statement of the proposition also as a map $\widehat{u}: \mathbb{R} \rightarrow \mathbb{L}_{\tau}^{h}\left(T^{*} \mathbb{T}^{2 N}, \sigma\right)$ as in (3.15). Thus $\widehat{u}$ is a flow line of $\mathcal{A}_{\sigma_{s}^{j}, V_{s}^{j}}$ :

$$
\partial_{s} \widehat{u}+\nabla_{J_{t}} \mathcal{A}_{\sigma_{s}^{j}, V_{s}^{j}}(\widehat{u}(s))=0 .
$$

Set

$$
\Delta(u):=\int_{-\infty}^{\infty}\left|\left(\frac{\partial}{\partial s} \mathcal{A}_{\sigma_{s}^{j}, V_{s}^{j}}\right)(\widehat{u}(s))\right| d s .
$$

We first bound $|\Delta(u)|$ in terms of $\left\|\partial_{s} u\right\|_{2}^{2}$. Note that

$$
\left|\left(\frac{\partial}{\partial s} \mathcal{A}_{\sigma_{s}^{j}, V_{s}^{j}}\right)(\widehat{u}(s))\right| \leq\left|\int_{0}^{\tau}\left(\frac{\partial}{\partial s} V_{s}^{j}\right)(u(s, \cdot)) d t\right|+\left|\left(\frac{\partial}{\partial s} \mathcal{A}_{\sigma_{s}^{j}}\right)([x(s, \cdot), z(s)])\right| .
$$

We can estimate the first term via

$$
\left|\int_{0}^{\tau}\left(\frac{\partial}{\partial s} V_{s}^{j}\right)(u(s, \cdot)) d t\right| \leq 2 \delta \tau\left\|V_{1}-V_{0}\right\|_{\infty} .
$$

For the second term we have

$$
\begin{aligned}
\left|\left(\frac{\partial}{\partial s} \mathcal{A}_{\sigma_{s}^{j}}\right)([x(s, \cdot), z(s)])\right| & \leq 2 \delta\left|\int_{\mathbb{S}_{\tau}} x(s, \cdot)^{*} \theta\right|+2 \delta\left|\int_{S^{1}} \gamma_{h}^{*} \theta\right| \\
& \leq 2 \delta\|\theta\|_{\infty}\left\|\partial_{t} x(s, \cdot)\right\|_{1}+2 \delta\left|\int_{S^{1}} \gamma_{h}^{*} \theta\right|,
\end{aligned}
$$


where the reference loops $\gamma_{h}$ were defined in (3.2) and

$$
\left\|\partial_{t} x(s, \cdot)\right\|_{1}:=\int_{0}^{\tau}\left|\partial_{t} x(s, \cdot)\right| d t .
$$

We now estimate

$$
\begin{aligned}
\left\|\partial_{t} x(s, \cdot)\right\|_{1} & \leq \sqrt{\tau}\left\|\partial_{t} x(s, \cdot)\right\|_{2} \\
& \leq \sqrt{\tau}\left(1+\left\|\partial_{t} x(s, \cdot)\right\|_{2}^{2}\right) \\
& \leq \sqrt{\tau}\left(1+\sup _{t \in \mathbb{S}_{\tau}}\left\|J_{t}\right\|_{\infty}^{2}\left\|\partial_{s} u(s, \cdot)\right\|_{2}^{2}+\|p(s, \cdot)\|_{2}^{2}\right),
\end{aligned}
$$

where the last line follows from taking horizontal components of the equation

$$
\partial_{t} u=J_{t}(u) \partial_{s} u+X_{\sigma_{s}^{j}, V_{s}^{j}}(t, u)
$$

(cf. (3.11)). Set

$$
\begin{gathered}
C_{1}:=2 \sqrt{\tau}\|\theta\|_{\infty} \\
C_{2}:=\sup _{t \in \mathbb{S}_{\tau}}\left\|J_{t}\right\|_{\infty}^{2}, \\
C_{3}:=2 \sqrt{\tau}\|\theta\|_{\infty}+2 \tau\left\|V_{1}-V_{0}\right\|_{\infty}+2\left|\int_{S^{1}} \gamma_{h}^{*} \theta\right| .
\end{gathered}
$$

We have shown

$$
\Delta(u) \leq C_{1} \delta\left\|\left.p\right|_{[0,1] \times \mathbb{S}_{\tau}}\right\|_{2}^{2}+C_{1} C_{2} \delta\left\|\partial_{s} u\right\|_{2}^{2}+C_{3} \delta .
$$

It follows from Lemma 3.5 that there exists a constant $T>0$ such that

$$
\left\|\left.p\right|_{[0,1] \times \mathbb{S}_{\tau}}\right\| \leq T\left(1+\left\|\left.\partial_{s} u\right|_{[0,1] \times \mathbb{S}_{\tau}}\right\|\right)
$$

(cf. Lemma 3.10), and hence

$$
\left\|\left.p\right|_{[0,1] \times \mathbb{S}_{\tau}}\right\|^{2} \leq 3 T^{2}\left(1+\left\|\left.\partial_{s} u\right|_{[0,1] \times \mathbb{S}_{\tau}}\right\|^{2}\right) .
$$

and hence we can estimate

$$
\Delta(u) \leq\left(3 C_{1} T^{2}+C_{1} C_{2}\right) \delta\left\|\partial_{s} u\right\|_{2}^{2}+\left(3 C_{1} T^{2}+C_{3}\right) \delta .
$$

Next, the conclusion of Lemma 3.9 becomes:

$$
\left\|\partial_{s} u\right\|_{2}^{2} \leq 4 \sup _{t \in \mathbb{S}_{\tau}} \frac{1}{\kappa_{J_{t}}}(E+\Delta(u)),
$$

where as $\kappa_{J_{t}}$ was defined in Lemma 2.3. Thus provided we choose $\delta$ small enough such that

$$
4 \sup _{t \in \mathbb{S}_{\tau}} \frac{1}{\kappa_{J_{t}}}\left(3 C_{1} T^{2}+C_{1} C_{2}\right) \delta<\frac{1}{2}
$$

we obtain

$$
\left\|\partial_{s} u\right\|_{2}^{2} \leq 8 \sup _{t \in \mathbb{S}_{\tau}} \frac{1}{\kappa_{J_{t}}}\left(E+\left(3 C_{1} T^{2}+C_{3}\right) \delta\right) .
$$

This completes the proof.

Proposition 3.14 is precisely what is needed in order to extend Theorem 3.11 to $s$-dependent trajectories (see [AS06, Lemma 1.21] - in particular the statements of Claim 1' and Claim 2'). One now applies a standard adiabatic argument to complete the proof of Theorem 3.13; see for instance [Sa199] for more details. 


\section{COMPUTING THE NOVIKOV FLOER HOMOLOGY}

Having defined the Floer homology $H F_{*}^{h}(\sigma, V, \tau)$ for any magnetic field $\sigma$ which is nonresonant in period $\tau$, and any potential $V$ (if $(\sigma, V, \tau)$ is degenerate then one first perturbs $V$, as in Remark 3.12), we now proceed to compute it.

Suppose we work on $\mathbb{T}^{2}$ with $\sigma=a_{0} \mu$ for some $a_{0} \in \mathbb{R}$ such that $a_{0} \tau \notin 2 \pi \mathbb{Z}$. By Remark 3.8 one has $\mathcal{P}_{\tau}^{h}(\sigma, 0)=\emptyset$ whenever $h \neq 0$, and for $h=0$ one has $\mathcal{P}_{\tau}^{h}(\sigma, 0) \cong \mathbb{T}^{2}$. This immediately implies that $H F_{*}^{h}(\sigma, 0, \tau)=0$ for $h \neq 0$, since in this case $(\sigma, 0, \tau)$ is vacuously non-degenerate when restricted to non-contractible critical points. In the contractible component $(\sigma, 0, \tau)$ is not non-degenerate on the contractible component, since the critical set is diffeomorphic to $\mathbb{T}^{2}$. So far if we wanted to compute $H F_{*}^{0}(\sigma, 0, \tau)$ we would first choose a small perturbation $V^{\varepsilon}$ and then define

$$
H F_{*}^{0}(\sigma, 0, \tau) \stackrel{\text { def }}{=} H F_{*}^{0}\left(\sigma, V^{\varepsilon}, \tau\right) .
$$

However there is a much easier method. Indeed, whilst $\mathcal{A}_{\sigma, 0}$ is not a Morse function on $\mathcal{L}_{\tau}^{0} T^{*} \mathbb{T}^{2}$, it is a Morse-Bott function. This gives an alternative way to compute $H F_{*}^{0}(\sigma, 0, \tau)$. One first picks an additional Morse function $f$ of the critical point set (in this case, a Morse function $f$ on $\mathbb{T}^{2}$, and then counts gradient flow lines with cascades of the pair $\left(\mathcal{A}_{\sigma, 0}, f\right)$. We emphasize that this is a particularly simple instance of Morse-Bott Floer homology, since there the critical manifold is connected. The correct grading to assign in the Morse-Bott case is given by

$$
\mu_{f}(w):=\mu_{\mathrm{CZ}}(w)-\frac{1}{2} \operatorname{dim}_{w} \operatorname{Crit}\left(\mathcal{A}_{a \mu, 0}\right)+\operatorname{ind}_{f}(w), \quad \text { for } w \in \operatorname{Crit}(f) \subset \operatorname{Crit}\left(\mathcal{A}_{a \mu, 0}\right),
$$

where $\operatorname{dim}_{w} \operatorname{Crit}\left(\mathcal{A}_{a \mu, 0}\right)$ denotes the local dimension of $\operatorname{Crit}\left(\mathcal{A}_{a \mu, 0}\right)$ at $w$, and ind $\operatorname{din}_{f}(w) \operatorname{denotes}$ the Morse index of $w$ as a critical point of $f$. In our case this simplifies to

$$
\mu_{f}(w)=\mu_{0}-1+\operatorname{ind}_{f}(w),
$$

where $\mu_{0} \in \mathbb{Z}$ is the common value

$$
\mu_{0}=\mu_{\mathrm{CZ}}(\text { constant loop }) .
$$

Anyway, this gives a new Floer homology group $H F_{*}^{0}(\sigma, 0, \tau)^{\mathrm{MB}}$, which does not depend on the choice of Morse function. Moreover for any sufficiently small perturbation $V^{\varepsilon}$, one has

$$
H F_{*}^{0}(\sigma, 0, \tau)^{\mathrm{MB}} \cong H F_{*}^{0}\left(\sigma, V^{\varepsilon}, \tau\right) .
$$

We refer the reader to [Fra04, Appendix A] or [BF11, Section 2.3] for more information. Since the critical manifold is simply $\mathbb{T}^{2}$ in our case, we immediately obtain

$$
H F_{*}^{0}(\sigma, 0, \tau) \cong H_{*+\mu_{0}-1}\left(\mathbb{T}^{2} ; \mathbb{Z}_{2}\right)
$$

where $\mu_{0}$ was defined in (4.2). In Lemma 5.4 below we show that $\mu_{0}=2 k+1$ where $k$ is the unique integer such that $2 \pi k<\left|a_{0}\right| \tau<2 \pi(k+1)$.

Now suppose $\sigma$ is non-resonant in period $\tau$ and $V$ is any potential such that $(\sigma, V, \tau)$ is nondegenerate. Let $a_{0}:=\int_{\mathbb{T}^{2}} \sigma$. Then $a_{0} \mu-\sigma$ is exact, say $a_{0} \mu-\sigma=d \theta$. Thus if we set $\sigma_{s}:=\sigma+s d \theta$, and we choose a generic homotopy from $V_{s}$ from $V=V_{0}$ to $V^{\varepsilon}=V_{1}$, we can apply Theorem 3.13, together with (4.1) and (4.3), to deduce that

$$
H F_{*}^{h}(\sigma, V, \tau) \cong H F_{*}^{h}\left(a_{0} \mu, 0, \tau\right) \cong\left\{\begin{array}{ll}
H_{*+2 k}\left(\mathbb{T}^{2} ; \mathbb{Z}\right), & h=0, \\
0, & h \neq 0,
\end{array},\right.
$$


where as before $k$ is the unique integer such that $2 \pi k<\left|a_{0}\right| \tau<2 \pi(k+1)$. Theorems A and $\mathrm{B}$ now follow by standard arguments. Indeed, if $(\sigma, V, \tau)$ is non-degenerate then since $\operatorname{rank} H F_{*}^{0}(\sigma, V, \tau)=\operatorname{rank} H_{*+2 k}\left(\mathbb{T}^{2} ; \mathbb{Z}_{2}\right)=4$, we immediately see that $\# \mathcal{P}_{\tau}^{0}(\sigma, V)$ is at least four. The argument is more involved in the degenerate case, but standard; see for instance [LO96] or [AH13].

Finally to deal with the case $N>1$ we first argue as above to see that if $\sigma=\sum_{j=1}^{N} \mathrm{p}_{j}^{*}\left(a_{j} \mu\right)$ for constants $a_{j} \in \mathbb{R}$ such that $a_{j} \tau \notin 2 \pi \mathbb{Z}$ then

$$
H F_{*}^{h}(\sigma, 0, \tau)= \begin{cases}H_{*+2 k}\left(\mathbb{T}^{2 N} ; \mathbb{Z}\right), & h=0, \\ 0, & h \neq 0,\end{cases}
$$

where $k=\sum_{j=1}^{N} k_{j}$ and $k_{j}$ is the unique integer such that $2 \pi k_{j}<\left|a_{j}\right| \tau<2 \pi\left(k_{j}+1\right)$. Then in the general case where $\sigma=\sum_{j=1}^{N} \mathbf{p}_{j}^{*} \sigma_{j}$, with each $\sigma_{j} \in \Omega^{2}\left(\mathbb{T}^{2}\right)$ non-resonant, we use the exact non-resonant deformation $\sigma_{s}:=\sigma+s d \theta$, where $\theta=\sum_{j=1}^{N} \mathbf{p}_{j}^{*} \theta$, and $\theta_{j} 1$-form on $\mathbb{T}^{2 N}$ satisfying

$$
d \theta_{j}=\left(\int_{\mathbb{T}^{2}} \sigma_{j}\right) \mu-\sigma_{j}
$$

Now Theorems A+ and B+ follow similarly.

\section{THE LAGRANGIAN SETTING}

In this section we briefly outline the "Lagrangian" method, with the aim of explaining Remark 1.4 from the Introduction. In this setting rather than working with the free loop space $\mathcal{L}_{\tau} \mathbb{T}^{2 N}$ we work with its Sobolev completion $\widetilde{\mathcal{L}}_{\tau} \mathbb{T}^{2 N}:=W^{1,2}\left(\mathbb{S}_{\tau}, \mathbb{T}^{2 N}\right)$. Unlike $\mathcal{L}_{\tau} \mathbb{T}^{2 N}$, the space $\widetilde{\mathcal{L}}_{\tau} \mathbb{T}^{2 N}$ carries the structure of a Hilbert manifold, and therefore is much better suited for doing Morse homology. In this section we use the Hilbert product $\langle\cdot, \cdot\rangle_{1,2}$ on $\widetilde{\mathcal{L}}_{\tau} \mathbb{T}^{2 N}$ defined by

$$
\langle\xi, \zeta\rangle_{1,2}:=\int_{0}^{\tau}\langle\xi(t), \zeta(t)\rangle d t+\int_{0}^{\tau}\left\langle\nabla_{t} \xi, \nabla_{t} \zeta\right\rangle d t
$$

We restrict our attention to the contractible component $\widetilde{\mathcal{L}}_{\tau}^{0} \mathbb{T}^{2 N}$; thus the functional $\mathcal{A}_{\sigma}$ from (3.1) is well defined on $\widetilde{\mathcal{L}}_{\tau}^{0} \mathbb{T}^{2 N}$ itself (cf. Remark 3.1). As before fix a time dependent potential $V \in C^{\infty}\left(\mathbb{S}_{\tau} \times \mathbb{T}^{2 N}, \mathbb{R}\right)$ and set

$$
L_{V}(t, x, v):=\frac{1}{2}|v|^{2}-V(t, x) .
$$

The Lagrangian action functional $\mathcal{S}_{\sigma, V}$ is defined as the sum

$$
\mathcal{S}_{\sigma, V}(\gamma):=\mathcal{S}_{V}(\gamma)+\mathcal{A}_{\sigma}(\gamma),
$$

where $\mathcal{S}_{V}$ is the standard Lagrangian action functional

$$
\mathcal{S}_{V}(\gamma):=\int_{0}^{\tau} L_{V}\left(t, \gamma(t), \partial_{t} \gamma(t)\right) d t
$$

The following lemma is straightforward.

Lemma 5.1. A loop $\gamma \in \widetilde{\mathcal{L}}_{\tau}^{0} \mathbb{T}^{2 N}$ is a critical point of $\mathcal{S}_{\sigma, V}$ if and only if there exists $w \in \mathcal{P}_{\tau}^{0}(\sigma, V)$ such that $\pi \circ w=\gamma$.

Recall that a $C^{1}$-functional $\mathcal{S}: \mathcal{M} \rightarrow \mathbb{R}$ on a Riemannian Hilbert manifold $\mathcal{M}$ satisfies the Palais-Smale condition if every sequence $\left(x_{k}\right) \subseteq \mathcal{M}$ for which $\mathcal{S}\left(x_{k}\right)$ is bounded and $\left\|d \mathcal{S}\left(x_{k}\right)\right\| \rightarrow$ 
0 admits a convergent subsequence (here $\|\cdot\|$ denotes the dual norm on $T_{x_{k}}^{*} \mathcal{M}$ ). We wish to prove:

Theorem 5.2. Suppose $\sigma$ is non-resonant in period $\tau$. Then for any potential $V \in C^{\infty}\left(\mathbb{S}_{\tau} \times \mathbb{T}^{2 N}, \mathbb{R}\right)$, the functional $\mathcal{S}_{\sigma, V}$ satisfies the Palais-Smale condition.

To see this note that

$$
d \mathcal{S}_{\sigma, V}(\gamma)[\xi]=\int_{0}^{\tau}\left\langle-\nabla_{t} \partial_{t} \gamma-Y_{\sigma}(\gamma) \partial_{t} \gamma-\nabla V_{t}(\gamma), \xi\right\rangle d t
$$

and hence if $\left(\gamma_{k}\right)$ is a sequence such that $\left\|d \mathcal{S}_{\sigma, V}\left(\gamma_{k}\right)\right\|=o(1)$ then

$$
\int_{0}^{\tau}\left|\nabla_{t} \partial_{t} \gamma_{k}+Y_{\sigma}\left(\gamma_{k}\right) \partial_{t} \gamma_{k}+\nabla V_{t}\left(\gamma_{k}\right)\right|^{2} d t=o(1) \text {. }
$$

Thus by an argument very similar to Lemma 3.5 one has that

$$
\int_{0}^{\tau}\left|\partial_{t} \gamma_{k}\right|^{2} d t=O(1)
$$

Now the standard argument, which was originally due to Benci [Ben86], goes through, as explained in [FMP12, Appendix A].

Ideally one would like to use $\mathcal{S}_{\sigma, V}$ to define a Morse complex $C M_{*}^{0}(\sigma, V, \tau)$ whose generators are the critical points of $\mathcal{S}_{\sigma, V}$. The homology of this complex should compute the singular homology of the space $\widetilde{\mathcal{L}}_{\tau}^{0} \mathbb{T}^{2 N}$. Moreover one expects that when defined, the homology $H M_{*}^{0}(\sigma, V, \tau)$ should be isomorphic to the Floer homology $H F_{*}^{0}(\sigma, V, \tau)$. In our setting such a construction is possible if and only if the functional $\mathcal{S}_{\sigma, V}$ is bounded below. We refer the reader to [AM06] for more information on the Morse complex, and to [AS06] for the idea behind the isomorphism between the Morse and Floer homologies. Here we note only the following point.

Lemma 5.3. Take $N=1$ and $\sigma=a \mu$ for some $a \in \mathbb{R}$. Assume that $a \tau \notin 2 \pi \mathbb{Z}$, and consider the functional $\mathcal{S}_{a \mu, V}: \widetilde{\mathcal{L}}_{\tau}^{0} \mathbb{T}^{2 N} \rightarrow \mathbb{R}$. Then the functional $\mathcal{S}_{a \mu, V}$ is bounded below if and only if $|a \tau|<2 \pi$.

Proof. The fact that $\mathcal{S}_{a \mu, V}$ is bounded below for $|a \tau|<2 \pi$ was proved in [FMP12, Appendix A]. Let us show that $\mathcal{S}_{a \mu, V}$ is not bounded below if $|a \tau|>2 \pi$. It suffices to consider the case $V=0$. Let $\widetilde{\gamma}_{R}: \mathbb{S}_{\tau} \rightarrow \mathbb{R}^{2 N}$ denote a circle of radius $R$ with centre the origin (explicitly $\left.\widetilde{\gamma}_{R}(t):=R e^{2 \pi i t / \tau}\right)$, and let $\gamma_{R}:=\mathrm{q} \circ \widetilde{\gamma}_{R}$, where $\mathrm{q}: \mathbb{R}^{2 N} \rightarrow \mathbb{T}^{2 N}$ denotes the projection map of the universal cover. Then

$$
\mathcal{S}_{a \mu, 0}\left(\gamma_{R}\right)=\frac{\pi R^{2}}{\tau}\left(\frac{2 \pi}{\tau}-a\right)
$$

which for $a \tau>2 \pi$ tends to $-\infty$ as $R \rightarrow \infty$ (if $a<0$ one should consider $t \mapsto \widetilde{\gamma}_{R}(\tau-t$ ) instead).

Finally, we compute the index jump $d$ referred to in (4.3).

Lemma 5.4. Take $N=1$ and $\sigma=a \mu$ for some $a \in \mathbb{R}$. Assume that $a \tau \notin 2 \pi \mathbb{Z}$, and let $k_{0} \in \mathbb{Z}$ denote the unique integer such that

$$
2 \pi k_{0}<|a| \tau<2 \pi\left(k_{0}+1\right) .
$$

Consider the functional $\mathcal{A}_{a \mu, 0}: \mathcal{L}_{\tau}^{0} \mathbb{T}^{2} \rightarrow \mathbb{R}$. Then the Conley-Zehnder index $\mu_{0}$ of a constant solution is given by

$$
\mu_{0}=2 k_{0}+1
$$


Proof. For simplicity we take $\tau=1$ and assume $a>0$ in what follows. It is easier to make the computation on the Lagrangian side, and then use the classical result due originally to [Dui76] that relates the Conley-Zehnder index with the Morse index of the corresponding solution on the Lagrangian side.

Moreover to compute the Morse index it is convenient to use a Fourier expansion. If $\gamma \in$ $\mathcal{L}_{0} \mathbb{T}^{2}$ then we can write

$$
\gamma(t)=\sum_{k \in \mathbb{Z}} e^{2 \pi k t j} \gamma_{k}
$$

Then one easily checks that

$$
\mathcal{S}_{a \mu, 0}(\gamma)=\sum_{k \in \mathbb{Z}}\left(2 \pi^{2} k^{2}-a \pi k\right)\left|\gamma_{k}\right|^{2}
$$

From this we can read off that the Morse index of a constant solution is

$$
\text { ind }_{\mathcal{S}_{a \mu, 0}} \text { (constant) }=2 \cdot \#\{k \in \mathbb{Z} \mid(2 \pi k-a) k<0\}=2 k_{0},
$$

where the 2 comes from the fact that the Fourier series have complex coefficients. The relation between this Morse index and the Conley-Zehnder index is given by

$$
\mu_{\mathrm{CZ}}(w)=\operatorname{ind}_{\mathcal{S}_{a \mu, 0}}(\pi \circ w)+\frac{1}{2} \operatorname{dim}_{w} \operatorname{Crit}\left(\mathcal{A}_{a \mu, 0}\right)
$$

(see [APS08, Section 4] for a detailed proof of this result in the case $\sigma=0$, and see [Mer11, Theorem 4.4] for justification as to why this result still holds for non-zero $\sigma$ ). Thus we have

$$
\mu_{0}=2 k_{0}+1
$$

as claimed.

\section{REFERENCES}

[AH13] P. Albers and D. Hein, In preparation, 2013.

[AM06] A. Abbondandolo and P. Majer, Lectures on the Morse complex for infinite dimensional manifolds, Morse Theoretic Methods in Nonlinear Analysis and Symplectic Topology (P Biran, O. Cornea, and F. Lalonde, eds.), Nato Science Series II: Mathematics, Physics and Chemistry, vol. 217, Springer-Verlag, 2006, pp. 1-74.

[APS08] A. Abbondandolo, A. Portaluri, and M. Schwarz, The homology of path spaces and Floer homology with conormal boundary conditions, J. Fixed Point Theory Appl. 4 (2008), no. 2, 263-293.

[AS06] A. Abbondandolo and M. Schwarz, On the Floer homology of cotangent bundles, Comm. Pure Appl. Math. 59 (2006), 254-316.

[AS10] _ Floer homology of cotangent bundles and the loop product, Geometry and Topology 14 (2010), 15691722.

[Ben86] V. Benci, Periodic solutions of Lagrangian systems on a compact manifold, J. Diff. Eq. 63 (1986), 135-161.

[BF11] Y. Bae and U. Frauenfelder, Continuation homomorphism in Rabinowitz Floer homology for symplectic deformations, Math. Proc. Camb. Phil. Soc. 151 (2011), 471-502.

[Dui76] J. J. Duistermaat, On the Morse Index in Variational Calculus, Adv. Math. 21 (1976), 173-195.

[Far04] M. Farber, Topology of Closed One-Forms, Math. Surveys and Monographs, vol. 108, Amer. Math. Soc., 2004.

[FMP12] U. Frauenfelder, Will J. Merry, and G. P. Paternain, Floer homology for magnetic fields with at most linear growth on the universal cover, J. Funct. Anal. 262 (2012), 3062-3090.

[Fra04] U. Frauenfelder, The Arnold-Givental conjecture and moment Floer homology, Int. Math. Res. Not. 42 (2004), 2179-2269.

[HS95] H. Hofer and D. Salamon, Floer homology and Novikov rings, The Floer Memorial Volume (H. Hofer, C. Taubes, A. Weinstein, and E. Zehnder, eds.), Progress in Mathematics, vol. 133, 1995.

[LO96] H. V. Lê and K. Ono, Cup-length estimates for symplectic fixed points, Contact and symplectic geometry (Cambridge, 1994) (C. B. Thomas, ed.), Cambridge University Press, 1996, pp. 268-295. 
[Mer11] W. J. Merry, On the Rabinowitz Floer homology of twisted cotangent bundles, Calc. Var. Partial Differential Equations 42 (2011), no. 3-4, 355-404.

[Sa199] D. Salamon, Lectures on Floer Homology, Symplectic Geometry and Topology (Y. Eliashberg and L. Traynor, eds.), IAS/Park City Math. Series, vol. 7, Amer. Math. Soc., 1999, pp. 143-225.

[Web02] J. Weber, Perturbed closed geodesics are periodic orbits: Index and transversality, Math. Z. 241 (2002), 45-81.

Urs Frauenfelder, Department of Mathematics and Research Institute of Mathematics, Seoul NATiOnAl University SAN 56-1 SHinRim-DONG KWANAK-GU, SEOUl 151-747, Korea

E-mail address: frauenfesnu.ac.kr

Will J. MERRy, DePARTMENT OF MATHEMATICs, ETH ZÜRICH

E-mail address: merry@math.ethz.ch

Gabriel. P. Paternain, Department of Pure Mathematics and Mathematical Statistics, UniverSITY OF CAMBRIDGE, CAMBRIDGE CB3 0WB, ENGLAND

E-mail address: g.p.paternain@dpmms.cam.ac.uk 